\title{
Clinical characteristics and postcode analysis of all positive SARS-CoV-2 PCR swabs in a large district general hospital in April 2020
}

\author{
Authors: Ahmad Shirazi-Nejad, ${ }^{\mathrm{A}}$ Ethar Abd Al-Shakour, ${ }^{\mathrm{A}}$ Tiwonge Nyirenda, ${ }^{\mathrm{A}}$ Mina Soliman ${ }^{\mathrm{A}}$ and Elmuhtady Said ${ }^{\mathrm{A}}$
}

\section{Introduction}

The aim of this study was to characterise the clinical course and delineate certain parameters of patients with positive COVID-19 PCR swab results recorded in our trust over a 1-month period at the peak of the pandemic. These include demographics, presenting symptoms, body mass index (BMI), comorbidities, mortality status, length of stay (LOS), requirement for non-invasive ventilation (NIV) / mechanical ventilation $(\mathrm{MV})$ and postcode analysis.

\section{Materials and methods}

Search criteria were defined within the Sunquest ICE system. All retrieved data were manually reviewed and positive results were tabulated. Certain demographic and clinical details were also obtained from VitalPAC systems. Google Maps was used to map out all the postcodes. Information for indices of deprivation was obtained from the UK government website.

\section{Results and discussion}

During April 2020, 528 individuals with positive COVID-19 PCR swab results were identified. 409 were members of the public and 119 were staff. The largest number of positive results was identified on 2 April $(n=36) .370$ patients lived in their own homes, 144 lived in a nursing/care home and 14 were receiving care in a hospice. The male:female ratio was $1: 1.3$, with an average age of 62.9 years (range $7-101$ years; mode 83 years). Of the 528 patients, 252 were admitted to hospital.

Common COVID-19 symptoms were identified and frequencies recorded (cough 118, shortness of breath (SOB) 116, pyrexia 103 , cough + SOB $56, \mathrm{SOB}+$ pyrexia 52 , cough+pyrexia 58 , cough $+\mathrm{SOB}+$ pyrexia 30 ). Important comorbidity frequencies were evaluated (cardiovascular disease 80, dementia 41, diabetes mellitus 39, respiratory illness 37, and active cancer / cancer treatment 17).

BMI was recorded in 166 patients (range $13.1-55.2 \mathrm{~kg} / \mathrm{m}^{2}$; median $26.3 \mathrm{~kg} / \mathrm{m}^{2}$ ). 128/166 of these patients were admitted to hospital and 23/128 were admitted to critical care for either
NIV or MV. The mean BMI of patients admitted to critical care was 31.83 , with an associated mortality of $34.78 \%$.

Of the 252 inpatients, 118 were discharged home alive (LOS range 1-76 days, $10 \mathrm{NIV}, 5 \mathrm{MV}$ ), but 134 died during their inpatient stay directly from COVID-19 or from associated sequelae (LOS range 1-57 days, $18 \mathrm{NIV}, 23 \mathrm{MV}$ ).

Barnsley is ranked 38/317 in the most deprived areas in England. We ascertained the results of 358 postcodes, all of which were in Barnsley or the surrounding areas and seven were outside South Yorkshire. Postcode S73 0HQ was identified as having the most cases (29) and includes two nursing homes. The top three postcodes are among the $30 \%$ most deprived neighbourhoods in the county. With regards to mortality, two of the top three postcodes are in the top 30\% most deprived neighbourhoods, but the second postcode with the most deaths is in the $40 \%$ least deprived neighbourhoods. This area also includes a care home. Interestingly, the postcodes inhabited by patients who survived with NIV or MV did not have multiple cases associated with a single postcode. It is likely that these patients were living in their own homes, rather than care facilities.

\section{Conclusion}

This study delineates various aspects of the clinical course of COVID-19. It is apparent that elevated BMI and postcode location as a surrogate marker for social deprivation increase the risk of severe disease.

\section{Conflicts of interest}

None declared. 